\title{
The severity of the articular deformities of the hands depending on the seropositivity in rheumatoid arthritis
}

${ }^{1}$ Faculty of Medicine, University „Ovidius” of Constanţa, Romania

${ }^{2}$ Balnear and Rehabilitation Sanatorium Techirghiol, Constanţa, Romania

${ }^{3}$ Clinical Rehabilitation Hospital, Eforie Nord, Romania

\begin{abstract}
Background. Rheumatoid arthritis (RA), met in the literature also as chronic evolving polyarthritis, is a chronic inflammatory disease, which typically involves the small and medium joints, with a symmetric distribution. Clinical studies shown that a proportion of patients with RA, without measurable rheumatoid factors are a distinct entity from those patients with seropositive RA. Around one third of patients with RA are seronegative for those two serological markers, the rheumatoid factors (RF) and anti-citrullinated peptide antibodies (Anti-CCP). Hands are the most frequently affected in both seropositive and seronegative RA and the first joints involved in the inflammatory process are the metacarpo-phalangeal joints, IFP joints and the radiocarpian joint. The big joints become later symptomatic, after the small joints are affected. Materials and methods: 66 patients files and the personal records of the patients consecutively admitted in the Rheumatology Department of the Clinical Emergency County Hospital Constanta. Patients from county Constanta were included in the study, with diagnosis of rheumatoid arthritis (diagnosis confirmed at least 2 years ago), including both forms (seropositive and seronegative) of the disease. The complete clinical exam was done for the patients included in the study, following the identification of the active joint disease. The mechanical status of the joint was observed by a single evaluator for each of those 66 patients with RA, with special focus on the presence of the deformities / ankyloses of the hands joints. Therefore, there were evaluated: the mobility (the number of the joints with limited movements).
\end{abstract} Elena Valentina Ionescu

Faculty of Medicine, University „Ovidius” of Constanta, Universitatii Alee No. 1, Campus B Constanţa, Romania

email : elena_valentina_ionescu@yahoo.com deformities, ankyloses (the number of the joints without mobility). The joint mobility was assessed by counting the hand joints with limited movements and the worsening of the prehension function. The presence of the following hand deformities was followed: fingers in "swan neck", "fusiform fingers", the thumb "in Z", and the aspect of "hand in the shape of a camel back". For evaluating the painful or swollen joints we used: the 28 joint index, the overall evaluation of the disease activity - by the patient (Patient Global Assessment-PGA) and by the doctor (Medical Doctor Global AssessmentMDGA), the score of disease activity DAS 28 (DAS28 - Disease Activity Score 28), the index of the clinical activity of the disease (Clinical Disease Activity IndexCDAI), as well as the simplified index of activity of the disease (Simplified Disease Activity Index-SDAI). It was determined for each patient the plasma RF and anticitrullinated peptide antibodies. Results and discussion. As expected, there is a bigger frequency of female patients in both forms of the disease (seropositive and seronegative) and regarding males the recurrence is approximate equal between those two groups. By comparing the frequency of the joint deformities at hand level between the 2 groups of patients included in the study (seropositive PR and seronegative PR), we saw a superiority of these (with all their forms) in the patients with seropositive polyarthritis. Conclusions. The clinical study done in this work is completing the data offered by the studies of some dedicated authors, who analysed the characteristic elements of rheumatoid arthritis depending on seropositivity. Our results confirm the idea that seronegative rheumatoid arthritis has a more gentle clinical evolution and is a less destructive form of disease.

Keywords: rheumatic polyarthritis, seropositive, seronegative, joint deformities 


\section{Introduction}

Rheumatoid arthritis is the most common joint inflammatory disease, affecting $0.5-1 \%$ of the world population. Although the prevalence is constant in the world, it can vary depending on the geographical location and of the race. The diagnosis of RA is based on the medical history and the clinical exam, laboratory tests and after other diagnoses were excluded [1,2]. Most of the patients have a chronic variable evolution of the disease, which untreated can give progressive, irreversible joint destruction, with permanent deformities, accompanied by functional deficit and reduction of life expectancy. The severity of the disease is expressed by the fact that more than $50 \%$ of patients stop their professional activity in the first 5 years of disease, and in 10\% appears a severe disability in the first 2 years of evolution. The appearance of some visceral lesions is responsible for the decrease of the life expectancy with 5 to 10 years. $\mathrm{RA}$ is representing not only an important medical problem, but also a social problem of public health. RA is extremely quirky regarding the severity and the evolution. The permanent remission can appear, but it is rare from the moment of the appearance of the joint disease [3].The clinical studies have shown the reduction of the life expectancy in patients with rheumatoid arthritis [4] with about 7 years in men and 3 years in women [3]. Other studies have proven that the severity markers of the disease like seropositivity [5] and the elevated titre of the sedimentation speed of the erythrocytes (VSH) [4], predict the mortality. The appearance of some visceral lesions is responsible of shortening the medium life expectancy with 5 to 10 years [6]. However, there had been proven that most of the patients with RA die because of cardiac and respiratory causes, common causes also in general population [3,7]. Due to its prevalence and the accessibility for laboratory investigations, RA served as a model for study of many inflammatory and immune-mediated diseases [8]. Clinical studies have suggested that a group of patients with RA, without expressing RF, represent a distinct clinical entity, than those with seropositive RA. Approximate one third of the patients with RA are seronegative for those 2 serological markers, the rheumatoid factor (FR) and antibodies against the cyclic citrulinated peptides. (Ac Anti-CCP). The rheumatoid factors, usually present as IgM-RF, but detectable in the group of patients also as IgG-RF and IgA-RF, are forming immune complexes which activate the complement, so that the vessel permeability increases, and the chemotactic factors are recruiting immuno-competent cells at joint level. The simple presence of the RF is insufficient for producing the arthritis, this being also identified also in infectious diseases, autoimmune diseases -others than RA, but also in $15 \%$ of the healthy population, especially in old people [9]. The sensibility and the specificity of the RF depend on the studied population, varying between $60-70 \%$ and respectively $50-90 \%$, its role in the aetiology of RA remaining unsure. Despite these, three quarters of the patients with RP are seropositive using the standard tests for the detection of the RF, or $90 \%$ of patients can be seropositive using the enzymelinked immunosorbent assays technique (ELISA) for the identification of the IgM-RF [10]. In 1998 there had been described for the first time a new type of antibodies which has been proved to have a high specificity ( $>95 \%)$ and sensibility $(80 \%)$ for RP. The discussion is about the antibodies against the cyclic citrulinated peptides (anti-CCP) with key role in the disease [11].

Multiple studies had shown that:

- There is a tight correlation between HLADR4 and the positivity of the anti-CCP;

- The citrulinated antigens are present in the inflamed synovial;

- The anti-CCP antibodies are locally produced at the synovial level, and the plasmocytes which secrete these antibodies have been isolated from patients with RA [12].

The quantitative determination of the anti-CCP antibodies is useful for the differential diagnosis of the inflammatory arthritis or other diseases which can clinically mimic RA and are associated with positive $\mathrm{RF}[11,12]$. More than this, the anti-CCP can precede the development of the joint signs and symptoms and have a predictive value for the negative prognosis of the disease, because big titres are associated with 
erosive disease [12].

\section{Materials and methods}

As work we used the patients files and personal medical documents, selecting 66 patients with RA from the Rheumatology Compartment of the Emergency Clinical County Hospital Constanta. The selected patients were re-evaluated regarding the validity of the diagnosis according to the ACR 1987 criteria, in this way the diagnosis established previously being confirmed as RA. This way, there had been included in the study patients from Constanta county, with certain rheumatoid polyarthritis diagnosis (diagnosis established at least 2 years ago), including both disease types: seronegative (group A) and seropositive (group $\mathrm{B})$. The patients which were diagnosed with chronic hepatitis with $\mathrm{B}$ or $\mathrm{C}$ virus were excluded (including those with undetectable viraemia), the patients with fibromyalgia, paraneoplazic syndromes, psoriasis, as well as patients with other chronical diseases which affect the joints (SEL, gout/pseudogout, sclerodermia, spondilartropathies, sarcoidosis, reactive arthritis or Lyme disease).

\section{Results and discussion}

By analysing the demographic data of the patients included in the study, there can be seen the dominance of the female patients in a percent of $89.39 \%$, male patients being represented only in the percent of $10.61 \%$. There is a greater frequency of the female gender in both disease forms (seropositive and seronegative), and regarding male patients the distribution is approximately equal between the 2 groups (Table I).
Table I - The gender distribution of the patients with seropositive and seronegative $R A$.

\begin{tabular}{|l|l|l|l|l|}
\hline Gender & Group A & Percent & Group B & Percent \\
\hline Male & 4 & 19.05 & 3 & 6.67 \\
\hline Female & 17 & 80.95 & 42 & 93.33 \\
\hline
\end{tabular}

The groups have been analysed regarding social integration and there can be seen significant results in RA seropositive group (B). The number of disabled patients and those retired on medical reasons is considerable higher in the seropositive group, the unemployment rate being similar. We observed the greater rate of employees in the seronegative RA patients group (Figures 1,2). The patients were registered as disabled by the local Commission of Disabled Persons.

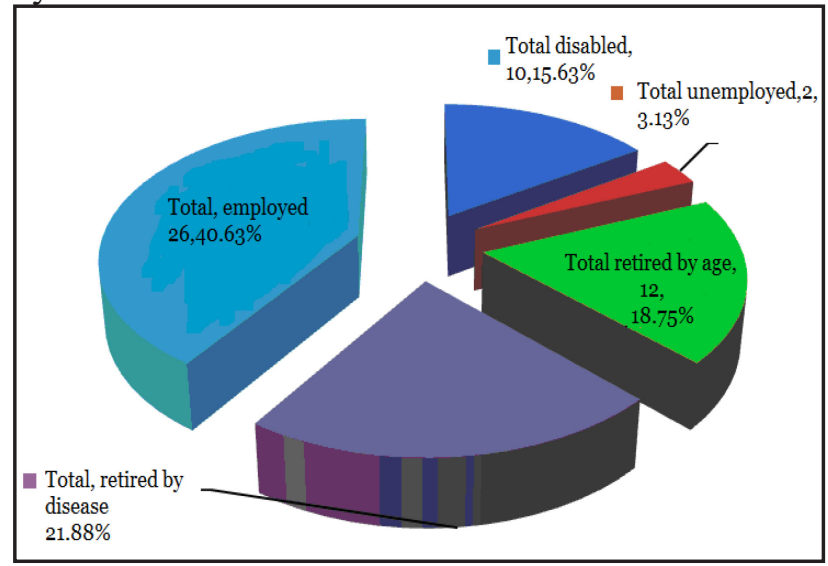

Figure 1 - Social integration of RA patients.

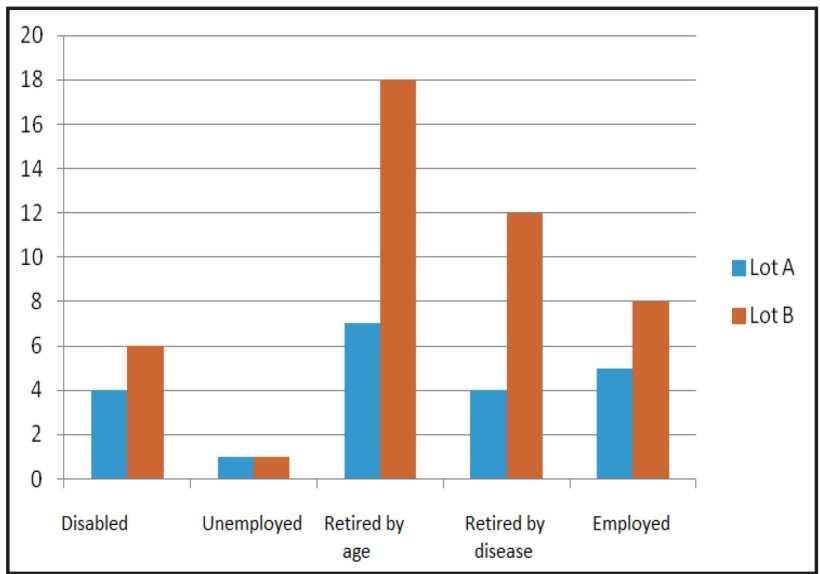

Figure 2 - Social integration of RA patients according to seropositive status (Group A-seropositive; group Bseronegative). 
Rheumatoid syndrome defines from a clinical point of view the symmetric, recent onset of polyarticular damage that may be associated with the presence of rheumatoid factor. The first joints involved in the inflammatory process are the metacaorpophalangeal, proximal interphalangeal and the radiocarpian joints.

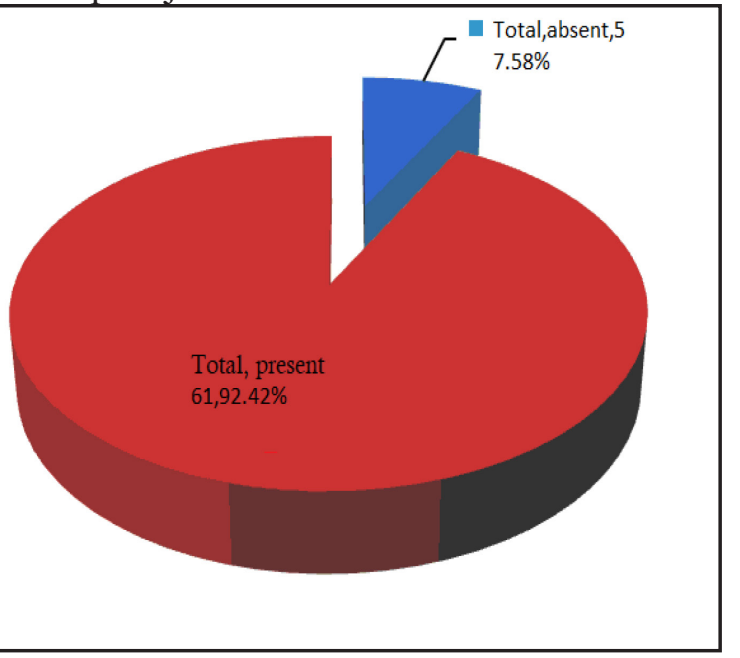

Figure 3 - Distribution of RA patients according to presence at onset of rheumatoid syndrome

According to medical history data, it appears that a significant percentage of the patients included in our study experienced rheumatoid syndrome at the onset of the disease $(92.42 \%$ versus $7.58 \%)$ (Tables II, III; Figure 3).

Table II - Distribution of RA patients according to presence at onset of rheumatoid syndrome.

\begin{tabular}{|l|c|c|c|c|c|}
\hline $\begin{array}{l}\text { Rheumatoid } \\
\text { syndrome at } \\
\text { onset }\end{array}$ & $\begin{array}{c}\text { Group } \\
\text { A }\end{array}$ & Percent & $\begin{array}{c}\text { Group } \\
\text { B }\end{array}$ & Percent & Total \\
\hline Absent & 4 & 19.05 & 1 & 2.22 & 5 \\
\hline Present & 17 & 80.95 & 44 & 97.78 & 61 \\
\hline Total & 21 & 100 & 45 & 100 & 66 \\
\hline
\end{tabular}

Differences were noted between the two groups in association of rheumatoid syndrome at onset. Thus, $97.78 \%$ of patients with RA, the seropositive group present this type of onset, compared to $80.95 \%$ of seronegative patients. The frequency of involvement at onset of metacarpophalangeal -MCF, proximal interphalangeal joints -PIF is $88.89 \%(n=40)$ of seropositive disease-associated RA and only $38.09 \%$ $(\mathrm{n}=8)$ in seronegative patients with this type of RA onset.

Table IV - Distribution of patients with seropositive and seronegative $R A$ in relation to joint damage.

\begin{tabular}{|l|c|c|c|c|c|}
\hline $\begin{array}{l}\geq 5 \text { swollen } \\
\text { joints at } \\
\text { the hands* }\end{array}$ & $\begin{array}{c}\text { Group } \\
\text { A }\end{array}$ & Percent & $\begin{array}{c}\text { Group } \\
\text { B }\end{array}$ & Percent & Total \\
\hline Absent & 16 & 76.19 & 30 & 66.67 & 46 \\
\hline Present & 5 & 23.81 & 15 & 33.33 & 20 \\
\hline Total & 21 & 100 & 45 & 100 & 66 \\
\hline
\end{tabular}

- metacarpophalangeal, proximal interphalangeal joints swollen at the time of evaluation

Table III - Characteristics of patients with seronegative RA versus seropositive RA

\begin{tabular}{|l|c|c|c|c|c|c|c|c|}
\hline Variable & \multicolumn{4}{|c|}{ Group A } & \multicolumn{4}{c|}{ Group B } \\
\hline & Average & SD & $\min$ & Max & Average & SD & min & Max \\
\hline DAS28 (VSH) & 4.37 & 1.50 & 1.89 & 6.91 & 4.32 & 1.62 & 2.02 & 7.40 \\
\hline DaS28 (PCR) & 3.74 & 1.55 & 1.48 & 6.72 & 3.68 & 1.57 & 1.31 & 6.75 \\
\hline Nb painful joints & 7.62 & 8.21 & 0 & 26 & 7.89 & 8.90 & 0 & 28 \\
\hline Nb swollen joints & 1.90 & 3.79 & 0 & 14 & 1.60 & 2.96 & 0 & 14 \\
\hline VAS & 47.14 & 25.50 & 10 & 90 & 42.89 & 25.19 & 10 & 90 \\
\hline CDAI & 18.95 & 14.48 & 2 & 46 & 18.09 & 14.67 & 2 & 50 \\
\hline SDAI & 27.03 & 18.21 & 5.2 & 70.5 & 33.74 & 35.81 & 3.6 & 197 \\
\hline HAQ & 2.82 & 1.47 & 1.1 & 7.1 & 2.66 & 1.46 & 1.05 & 8.12 \\
\hline Lee & 16.10 & 9.60 & 4 & 33 & 17.27 & 8.57 & 2 & 34 \\
\hline $\begin{array}{l}\text { Rheumatoid } \\
\text { factor }\end{array}$ & 7.73 & 1.55 & 5.6 & 12 & 132.58 & 299.96 & 6 & 1536 \\
\hline Ac anti-CCP & 1.81 & 0.75 & 1 & 4 & 645.77 & 1615.77 & 1.7 & 6577 \\
\hline
\end{tabular}


The results of clinical evaluation of the 66 RA patients were compared between the two groups (seropositive RA and seronegative RA). There were differences in the number of swollen joints at the time of evaluation; thus, $33.33 \%$ of patients with seropositive RA, presented at the time of evaluation a clinically active disease with at least 5 swollen joints in the hands compared to $23.81 \%$ of the seronegative patients. There is a higher incidence of patients with seronegative RA who have no swollen joints in the hands or have less than 5 synovites in MCF and PIF joints (76.19\% vs. 66.67\%) (Table IV, Figure 5).

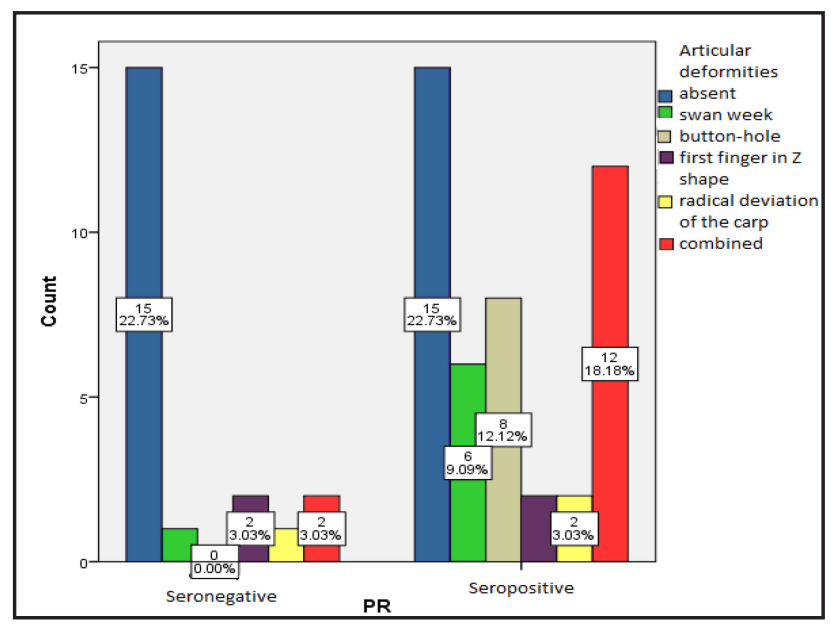

Figure 4 - Distribution of patients with seropositive and seronegative $R A$ depending on the type of joints deformities.

By analysing the frequency of hand deformities in the hands between the two patient groups included in the study (seropositive and seronegative RA), we found their superiority (in all their forms) among the patients with seropositive polyarthritis. Most often these occur in the combined version. There were no significant differences in the proportion of patients who had deformities in the "swan neck" or "buttonhole", incorrect ulnar deviation in the dominant or non-dominant hand. It seems that the middle finger and the index, from the dominant hand, exhibit more severe radiological changes (Figure 4).

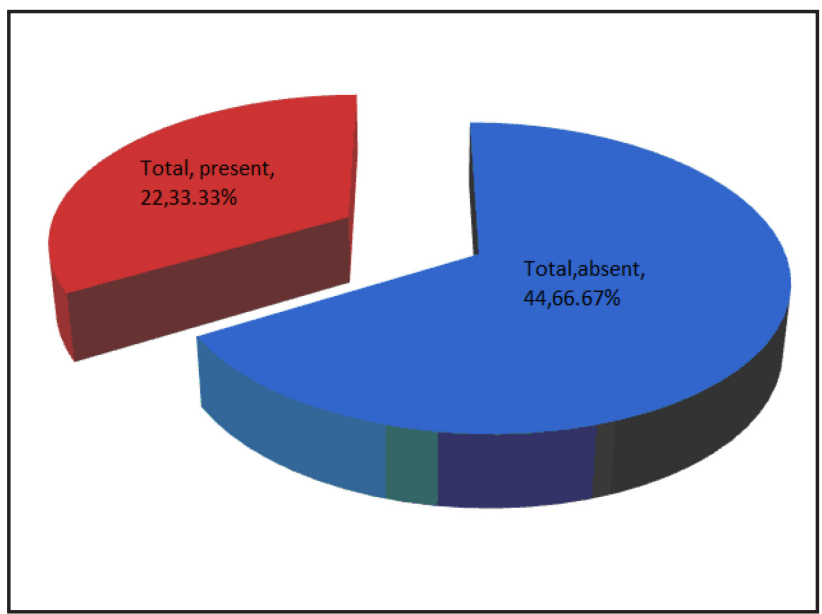

Figure 5 - The distribution of patients with seropositive and seronegative RA according to the association of clinically obvious joint ankyloses.

There were statistically significant differences between the two groups in terms of ankyloses evolution, which had a significantly increased frequency among patients with seropositive RA. For example, $40 \%$ of patients with seropositive PR have severe ankyloses, compared to only $19.05 \%$ of patients with seronegative disease.

\section{Conclusions}

Regarding the particularities of the group of patients with defined rheumatoid arthritis that have been included in this study, we can make the following statements:

- $97.78 \%$ of patients with seropositive polyarthritis associate rheumatoid arthritis at onset, compared to $80.95 \%$ of seronegative patients;

- MCF and PIF joints are co-affected at onset in $88.89 \%(n=40)$ of seropositive disease-associated RA and only $38.09 \%(n=8)$ of seronegative patients;

- $33.33 \%$ of patients with RA, the seropositive form present at the time of evaluation a form of clinically active disease with at least 5 swollen joints in the hands, compared with $23.81 \%$ of the 
seronegative patients;

- we have obtained results similar to those provided by the literature on the superiority of hand deformities in patients with seropositive disease;

- pain, reoccurrence and functional impotence are the main symptoms that characterize rheumatoid arthritis, constituting important causes of temporary or even definitive inability to work, with significant personal and social implications and costs.

The results of the clinical trial completed the data provided by the studies of established authors, which analysed the elements characteristic of rheumatoid arthritis according to seropositivity.

\section{References}

1. Aletaha, D. \& Smolen, J. (2005). The Simplified Disease Activity Index (SDAI) and the Clinical Disease Activity Index (CDAI): a review of their usefulness and validity in rheumatoid arthritis. Clinical and experimental rheumatology. 23(5), S100.

2. Andonopoulos, A.P., Drosos, A.A., Skopouli, F.N., Acritidis, N.C. \& Moutsopoulos, H.M. (1987). Secondary Sjogren's syndrome in rheumatoid arthritis. J Rheumatol. 14(6), 10981103.

3. Hakim, A., Clunie, G. \& Haq, I. (2008). Oxford handbook of rheumatology: OUP Oxford (pp 236).

4. Wolfe, F., Mitchell, D.M., Sibley, J.T., Fries, J.F., Bloch, D.A., Williams, C.A., Spitz, P.W., Haga, M., Kleinheksel, S.M. \& Cathey, M.A. (1994). The mortality of rheumatoid arthritis. Arthritis Rheum. 37(4), 481-494.

5. Mitchell, D.M., Spitz, P.W., Young, D.Y., Bloch, D.A., McShane, D.J. \& Fries, J.F. (1986). Survival, prognosis, and causes of death in rheumatoid arthritis. Arthritis \& Rheumatology. 29(6), 706-714.

6. Bolosiu, H., Ionescu, R., Chirieac, R., Georgescu, L., Rednic, S. \& Suta, M. (2011).
Ghidul de tratament al poliartriteireumatoide. RevistaRomană de Reumatologie. 20(1), 36-53

7. Raynauld, J.-P. (1997). Cardiovascular mortality in rheumatoid arthritis: how harmful are corticosteroids? Journal of rheumatology. 24(3), 415-416.

8. Firestein, G.S., Budd, R.C., Harris, E.D., McInnes, I.B., Ruddy, S. \& Sergent, J.S. (2009). Kelleys. Textbook of Rheumatology, Eighth Edition, (pp. 1035).

9. Klareskog, L., Padyukov, L., Rönnelid, J. \& Alfredsson, L. (2006). Genes, environment and immunity in the development of rheumatoid arthritis. Current opinion in immunology. 18(6), 650-655.

10. Khosla, P., Shankar, S. \& Duggal, L. (2004). Anti $\mathrm{CCP}$ antibodies in rheumatoid arthritis. $J$ Indian Rheumatol Assoc. 12(1), 143-146.

11. Pruijn, G.J., Vossenaar, E.R., Drijfhout, J.W., Van Venrooij, W.J. \& Zendman, A.J. (2005). Anti-CCP antibody detection facilitates early diagnosis and prognosis of rheumatoid arthritis. Current Rheumatology Reviews. 1(1), 1-7

12. Vossenaar, E.R. \& van Venrooij, W.J. (2004). Anti-CCP antibodies, a highly specific marker for (early) rheumatoid arthritis. Clinical and Applied Immunology Reviews. 4(4), 239-262. 